\title{
Pitfalls of Split-Night Polysomnography
}

\author{
Shrinivas Kambali MD, Gilbert Berdine MD
}

Sleep disordered breathing is a common problem. At least $10 \%$ of the population suffers from sleep disorders, and they are often related to other disorders, such as hypertension, cardiovascular disease, cerebrovascular disease, pulmonary hypertension, diabetes, metabolic syndrome, and depression. ${ }^{2}$ Polysomnograms (PSG) a real important tool for diagnosis of sleep apnea. However, patient access to complete diagnostic polysomnography followed by a second titration study may be limited due to costs. One alternative to a two study sequence is to use split-night polysomnography which includes both a diagnostic phase and positive pressure titration phase if obstructive sleep apnea is present during a single night study. We present two cases for whom splitnight polysomnography was used for diagnosis and treatment of the sleep apnea.

\section{Case1 - Figure1}
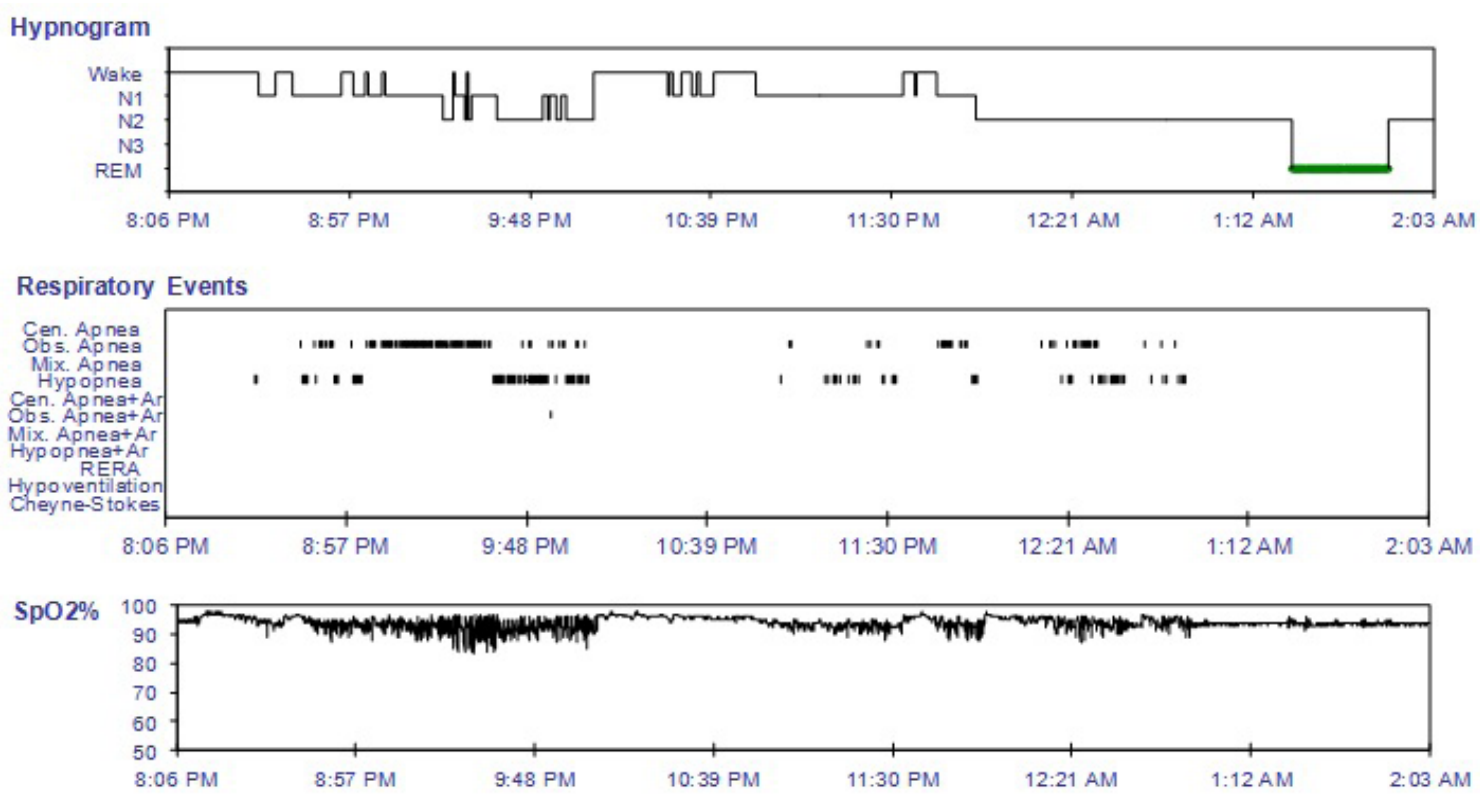

Body Position
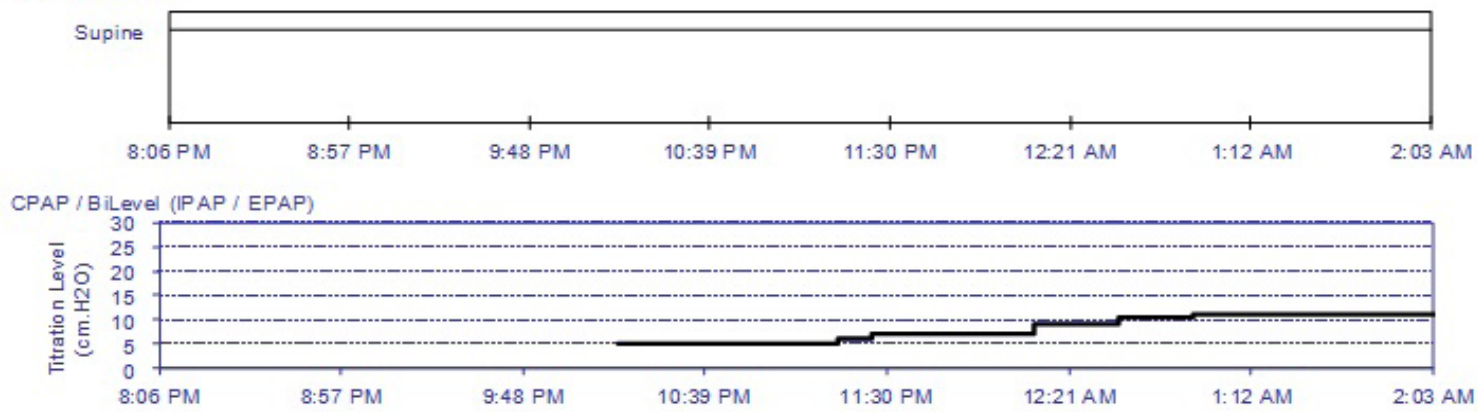
Figure 1 is a graphical summary of the sleep study, or hypnogram, for the first patient. PSG data from this patient included a total sleep time of 96.6 minutes, a sleep efficiency of $69 \%$, a sleep latency of 25.1 minutes, and an apnea-hypopnea index (AHI) of 80.7 events per hour. Rapid eye movement (REM) sleep was not observed during the diagnostic portion. There were episodes of oxygen desaturation with a mean saturation of $92.7 \%$ and a minimum saturation of $83 \%$. Continuous positive airway pressure
(CPAP) titration was started at $5 \mathrm{cmH}_{2} \mathrm{O}$ and gradually increased to $11 \mathrm{cmH}_{2} \mathrm{O}$. The AHIs were 12.8, 46.0, 29.4, 66.2, 34.4, and 0 using CPAP levels of 5, 6, 7, $8,9,10$, and 11 , respectively. There were no central apneas. During the final CPAP setting of $11 \mathrm{cmH}_{2} \mathrm{O}$, there were no apnea/hypopnea events, there were 27 minutes of REM sleep, and the mean oxygen saturation was $93 \%$ with a minimum saturation of $91 \%$. The overall interpretation was a diagnosis of OSA with a therapeutic recommendation for $11 \mathrm{cmH}_{2} \mathrm{O}$ CPAP.

\section{Case2 - Figure2}
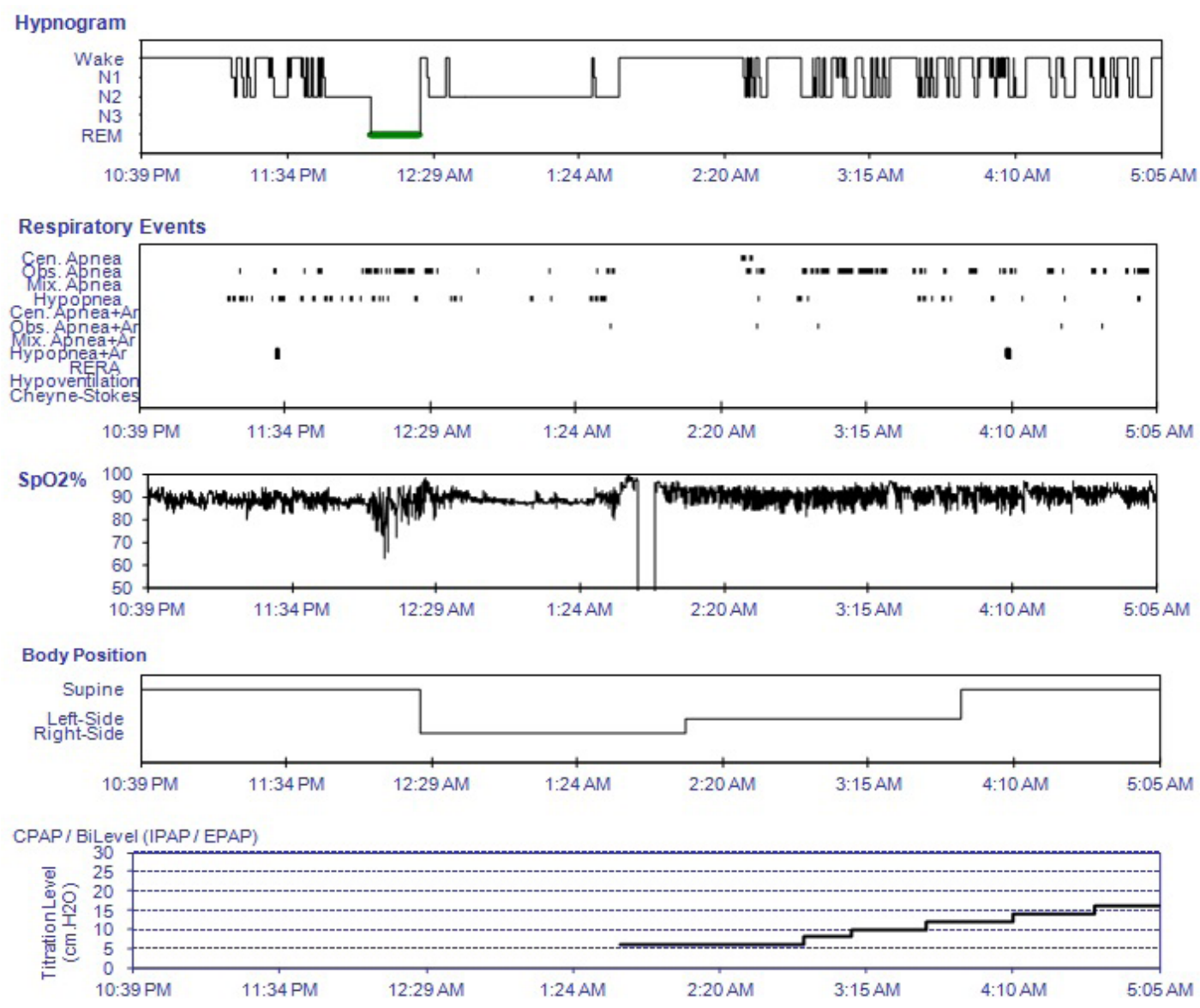

Figure 2 is the hypnogram for the second patient. PSG data included total sleep time of $147 \mathrm{~min}-$ utes, a sleep efficiency of $70.2 \%$, a sleep latency of 33.6 minutes, an AHI of 39.2, a REM AHI of 61.6, a mean oxygen saturation of $88.5 \%$, and a minimum oxygen saturation of $63 \%$. CPAP was initiated at 6 $\mathrm{cmH}_{2} \mathrm{O}$ and gradually increased to $16 \mathrm{cmH}_{2} \mathrm{O}$. The patient continued to have apnea/hypopnea events at all CPAP levels tested. AHIs were 102.6, 105.5, 117.5, 70.750 .6 , and 66.7 at CPAP levels of $6,8,10,12,14$, and 16 , respectively. The patient continued to have oxygen desaturation during the CPAP titration. There was no REM sleep throughout the titration study. 
Both patients had a significant number of events during the first two hours. Although the first patient had a higher number of events $(\mathrm{AHI}$ of $80.7 \mathrm{vs}$. 39.2), CPAP titration was successful with complete resolution of the apnea/hypopnea events using CPAP $11 \mathrm{cmH}_{2} \mathrm{O}$. The second patient had a lower $\mathrm{AHI}$, but CPAP titration was unsuccessful in this patient.

\section{Discussion}

Split-night sleep studies involve diagnostic polysomnography during the first portion of the night followed by CPAP titration for the remainder of the night. This approach has been used to diagnose sleep apnea and titrate CPAP during a single night and can reduce costs. McArdle et al reported that there was no difference in long term CPAP use, median nightly CPAP use, post-treatment Epworth scores, and the frequency of clinic visits in patients managed with split-night sleep studies compared to patients managed with standard full-night polysomnography. Median time from referral to treatment was less for the split-night patients than for full-night patients. ${ }^{4}$

Current American Academy of Sleep Medicine guidelines recommend that split-night polysomnography should be performed only if the $\mathrm{AHI}$ is 40 or greater during the first two hours of recording. Chou et al found that split-night sleep studies were effective for diagnosing severe OSA. The two hour PSG was as effective as both a 2.5 hour PSG and three hour PSG, and the two hour PSG was more effective than 1.5 hour-PSG. This suggests that the diagnostic portion of the sleep study should beat least two hours. Patients with an $\mathrm{AHI}$ less than 40 during the first two hours of sleep require a full-night diagnostic study and cannot be split. The CPAP titration portion of a split-night study should last at least three hours. PSG during the CPAP titration portion should demonstrate elimination of respiratory events during both REM and non-REM sleep. If this is not achieved, then a second full-night polysomnogram for CPAP titration should be performed. ${ }^{5}$ Yamashiro et al reported a study in 1995 that confirmed the effectiveness of CPAP titration using a split protocol. There was no significant difference between the split-night and two night strategies.
The final CPAP pressure was slightly lower at the end of the split-night study than at the end the full-night CPAP study.

Despite the promising advantages of splitnight PSG, there are concerns about using split-night studies as the preferred approach. One concern is the individual variability in the sleep pattern. Another concern is accurate assessment of the sleep architecture. Some patients demonstrate sleep disordered breathing only during REM sleep; a split-night study may have inadequate time for proper titration in these patients. Both of our cases were appropriate for splitnight protocol. The first patient had a successful CPAP titration during the first night, but the second patient required a second full-night study. Consequently, split-night PSGs are effective in some but not all patients. Furthermore, some patients who qualify for a split-night study cannot be adequately titrated without a full-night titration study.

\section{KEYPOINTS}

1. Split-night polysomnography provides enough diagnostic information and time for successful CPAP titration in some patients to avoid the need for a second night of polysomnography.

2. This approach is both cost effective and convenient for the patient.

3. Patients with successful split-night studies do as well as patients with two overnight studies.

Key Words : polysomnography, OSA, CPAP, titration

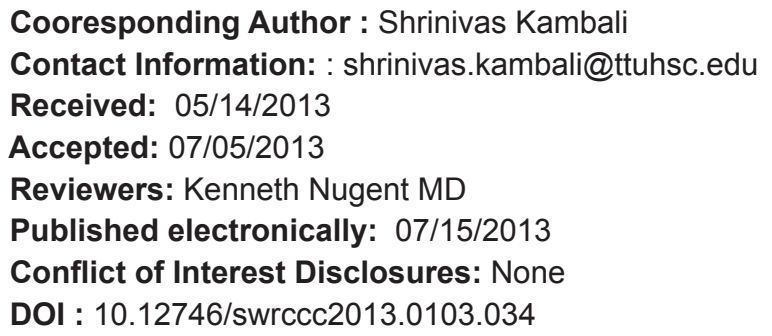

Cooresponding Author: Shrinivas Kambali

Contact Information: : shrinivas.kambali@ttuhsc.edu

Received: 05/14/2013

Accepted: 07/05/2013

Reviewers: Kenneth Nugent MD

Published electronically: 07/15/2013

Conflict of Interest Disclosures: None

DOI : 10.12746/swrccc2013.0103.034 


\section{REFERENCES}

1. Mason RJ, Broaddus VC, Martin TR, King TE, Schraufnagel DE, Murray JF, and NadelJA. Chapter 79, 1881-191. Murray and Nadel's Textbook of Respiratory Medicine, Fifth Edition

2. American Academy of Sleep Medicine. International classification of sleep disorders, 2nd ed: Diagnostic and coding manual, American Academy of Sleep Medicine, Westchester, IL 2005

3. Patel NP, Ahmed M, Rosen I. Split-night polysomnography. Chest. 2007; 132(5):1664-1671. doi:10.1378/chest.06-1801

4. McArdle N, Grove A, Devereux G, Mackay-Brown L, Mackay T, Douglas NJ. Split-night versus full-night studies for sleep apnoea/hypopnoea syndrome Eur Respir J. 2000 Apr; 15(4):670-5. 5. Kushida CA, Littner MR, Morgenthaler M, Alessi CA, Bailey D, Leman JC, Friedman L, Hirshkowitz M, Kapen S, Kramer M, Chiong TL, Loube DL, Owens J, Pancer JP, Wise M, Practice Parameters for the Indications for Polysomnography and Related Procedures: An Update for 2005.Sleep, vol. 28, no. 4, 2005 